VOL. 18 (1978), 65-75.

\title{
Duality in finite dimensional complex space
}

\section{C.H. Scott and T.R. Jefferson}

\begin{abstract}
The idea of duality is now a widely accepted and useful idea in the analysis of optimization problems posed in real finite dimensional vector spaces. Although similar ideas have filtered over to the analysis of optimization problems in complex space, these have mainly been concerned with problems of the linear and quadratic programing variety. In this paper we present a general duality theory for convex mathematical programs in finite dimensional complex space, and, by means of an example, show that this formulation captures all previous results in the area.
\end{abstract}

\section{Introduction}

Over the last decade, considerable effort has been directed towards optimization problems over a complex space. This work was initiated by Levinson [7] who extended some basic theorems of linear inequalities to complex space and derived a duality theory for complex linear programs. Subsequently, these results were extended to polyhedral cones in complex space by Ben-Israel [3] and Abrams and Ben-Israel [2]. Hanson and Mond [5], Mond [8], and Jain and Saxena [6] have analysed complex programs involving quadratic and square root functions. A general theory of duality, in the sense of Wolfe [11], has been given by Abrams [1].

Another approach to duality in mathematical programming based on the conjugate transform [4] has become established in the past few years. This has been exhaustively pursued in finite dimensional real space by Rockafellar [10] and Peterson [9]. Our purpose is to extend duality theory Received 14 October 1977. 
in the sense of Peterson to complex space. We obtain a completely symetric duality theory for convex programs in complex space. This duality theory has a great advantage over that of Wolfe in that the primal variables do not appear in the dual. As an example of the theory we consider a convex program with an objective function containing both differentiable and non differentiable parts subject to quadratic constraints. All previously studied programs in complex space as listed above are particular cases of this theory.

\section{Convexity}

DEFINITION 1. $S \subset C^{n}$ is a convex set if for all $x_{1}, x_{2} \in S$ and for all $\lambda$ with $0 \leq \lambda \leq 1$,

$$
\lambda x_{1}+(1-\lambda) x_{2} \in S .
$$

DEFINITION 2. $S \subset c^{n}$ is a convex cone if for all $x_{1}, x_{2} \in S$ and for all $\lambda_{1} \geq 0, \lambda_{2} \geq 0$,

$$
\lambda_{1} x_{1}+\lambda_{2} x_{2} \in S
$$

DEFINITION 3. $S^{*}$, the polar of a convex cone $S$, is

$$
\left\{y \in C^{n} \mid \operatorname{R\in }\langle y, x\rangle \geq 0 \text {, for all } x \in S\right\} \text {. }
$$

DEFINITION 4. A pair of function $g$ and convex set $G \subset c^{2 n},[g, G]$, is convex with respect to the closed convex cone $S$ if for any $z^{1}$ and $z^{2}$, and $0 \leq \lambda \leq 1$,

$$
\lambda g\left(z^{1}, \bar{z}^{-1}\right)+(1-\lambda) g\left(z^{2}, \bar{z}^{2}\right)-g\left(\lambda z^{1}+(1-\lambda) z^{2}, \lambda \bar{z}^{1}+(1-\lambda) \bar{z}^{2}\right) \in S,
$$

where $G \subset Q=\left\{\left(w^{1}, w^{2}\right) \in C^{2 n}\right\}, w^{2}=\bar{w}^{\perp}, g: G \rightarrow c^{m}$, and $s \subset C^{m}$.

DEFINITION 5. The pair [Re $g, G]$ is convex with respect to a closed convex cone $T$ in $R^{m}$ if, for any $z^{1}$ and $z^{2}$, and $0 \leq \lambda \leq 1$, $\lambda \operatorname{Re} g\left(z^{1}, \bar{z}^{-1}\right)+(1-\lambda) \operatorname{Re} g\left(z^{2}, \bar{z}^{-2}\right)-\operatorname{Re} g\left(\lambda z^{1}+(1-\lambda) z^{2}, \lambda \bar{z}^{1}+(1-\lambda) \bar{z}^{2}\right) \in T$, where $G \subset Q \subset C^{2 n}$ and $\operatorname{Re} g: G+R^{m}$. 
For the remainder of the paper we will consider $g$ such that Re $g: G \rightarrow R$ and $T=R_{+}$.

DEFINITION 6 . The convex function $[g(z, \bar{z}), G]$ convex with respect to convex cone $S$ is called closed if the set

$$
\{(z, \bar{z}, \alpha) \mid \alpha=g(z, \bar{z})+\beta, \beta \in S,(z, \bar{z}) \in G\}
$$

is closed. We shall assume all functions are closed.

If $g$ is convex with respect to $S$ and $\operatorname{Re} S=R_{+}$, then we can associate a set of dual points with each pair $\left(z_{0}, \bar{z}_{0}\right) \in G$ and convex function $\left[g: G \rightarrow C^{\mathcal{l}}\right]$, called the subgradient.

DEFINITION 7 . The subgradient set of $g(z, \bar{z})$ at $\left(z_{0}, \bar{z}_{0}\right)$ is defined by

$$
\left\{(y, \bar{y}) \mid \operatorname{Re} g\left(z_{0}, \bar{z}_{0}\right)-\operatorname{Re}\left\langle y, z_{0}-z\right\rangle \leq \operatorname{Re} g(z, \bar{z}) \text {, for all }(z, \bar{z}) \in G\right\} .
$$

We denote this by $\partial g\left(z_{0}, \bar{z}_{0}\right)$.

Moreover if $g$ is convex with respect to $S$ and $\operatorname{Re} S=R_{+}$we have the following definition of a convex conjugate transform for complex spaces. We will assume the condition that $\operatorname{Re} S=R_{+}$for the remainder of the paper.

DEFINITION 8. The conjugate transform of a function $g(z, \bar{z})$ defined on $(z, \bar{z}) \in G,[g(z, \bar{z}): G]$ is $[h(y, \bar{y}): H]$, where

$$
\operatorname{Re} h(y, \bar{y})=\sup _{(z, \bar{z}) \in G}[\operatorname{Re}(y, z)-\operatorname{Re} g(z, \bar{z})] .
$$

Im $h(y, \bar{y})$ is the complex conjugate function of $\operatorname{Re} h(y, \bar{y})$. Thus $h(y, \bar{y})$ is analytic in $(y, \bar{y})$. The complex conjugate is known up to a constant. For uniqueness choose some $(z, \bar{z}) \in G$ and $(y, \bar{y}) \in \partial g(z, \bar{z})$ and set

$$
h(y, \bar{y})=\langle y, z\rangle-g(z, \bar{z})
$$

and

$$
H=\left\{(y, \bar{y}) \mid \sup _{(z, \bar{z}) \in G} \operatorname{Re}(y, z)-\operatorname{Re} g(z, \bar{z})<\infty\right\} .
$$


The above gives rise to the conjugate inequality

$\operatorname{Re} g(z, \bar{z})+\operatorname{Re} h(y, \bar{y}) \geq \operatorname{Re}(y, z\rangle$ for $(z, \bar{z}) \in G,(y, \bar{y}) \in H$. This is satisfied at equality for

$$
(y, \bar{y}) \in \partial g(z, \bar{z}),
$$

or equivalently

$$
(z, \bar{z}) \in \partial h(y, \bar{y})
$$

DEFINITION 9. The positive homogeneous extension of a convex function $h$ is $\left[h^{+}(y, \bar{y}, \lambda), H^{+}\right]$where $\operatorname{Re} h^{+}(y, \bar{y}, \lambda)=\left\{\begin{array}{l}\sup _{(z, \bar{z}) \in G} \operatorname{Re}\langle y, z\rangle \text { if } \lambda=0 \text { and } \sup _{(z, \bar{z}) \in G} \operatorname{Re}\langle y, z\rangle\langle\infty, \\ \operatorname{Re} \lambda h(y / \lambda, \bar{y} / \lambda) \text { if } \lambda \in S^{*} \backslash\{0\} \text { and }(y / \lambda, \bar{y} / \lambda) \in H,\end{array}\right.$ $H^{+}=\left\{(y, \bar{y}, \lambda) \mid \sup _{(z, \bar{z}) \in G} \operatorname{Re}\langle y, z\rangle<\infty, \lambda=0\right\}$

$$
\cup\left\{(y, \bar{y}, \lambda) \mid(y / \lambda, \bar{y} / \bar{\lambda}) \in H, \lambda \in S^{*} \backslash\{0\}\right\},
$$

$S^{*}$ is the polar of $S$, the set with respect to which $g$ is convex.

The conjugate transform and its positive homogeneous extension are both closed convex functions by construction and the properties of analytic functions.

If $[\operatorname{Re} g(z, \bar{z}), G]$ and $[\operatorname{Re} h(y, \bar{y}), H]$ are closed conjugate functions, we have by construction that

$$
\operatorname{Re} g(z, \bar{z})+\operatorname{Re} h(y, \bar{y}) \geq \operatorname{Re}\langle y, z\rangle
$$

for $(z, \bar{z}) \in G,(y, \bar{y}) \in H$.

The following two lemmas are generalisations of Peterson's work [9] to complex space.

LEMMA 1. The conjugate transform of $\left[\operatorname{Re} g^{+}(z, \bar{z}, k): \kappa G\right]$ for fixed $k \in U^{*} \backslash\{0\}$ is $[\operatorname{Re} \kappa h(y, \bar{y}): H]$, where $g$ takes values over $U$.

Proof. By definition, the conjugate transform of $\left[\operatorname{Re} g^{+}(z, \bar{z}, \kappa): \kappa G\right]$ for fixed $\kappa \in U^{*} \backslash\{0\}$ is given by

$$
\begin{aligned}
\sup _{(z, \bar{z}) \in K G} & {\left[\operatorname{Re}\langle y, z\rangle-\operatorname{Re} g^{+}(z, \bar{z}, k)\right] } \\
= & \sup _{(z / K, \bar{z} / \bar{K}) \in G}[\operatorname{Re}\langle y, z\rangle-\operatorname{Re} k g(z / k, \bar{z} / \bar{k})], \text { since } \kappa \in U^{*} \backslash\{0\} .
\end{aligned}
$$


Substituting for the inner product and using Definition 8 , equation ( 1 ) gives

$$
\operatorname{Re} \kappa h(y, \bar{y}) \text { with }(y, \bar{y}) \in H .
$$

LEMMA 2. The conjugate transform of $\left[\operatorname{Re} g^{+}(z, \bar{z}, K): \dot{G}^{+}\right]$is the identically zero function on

$$
\{(y, \bar{y}) \mid(y, \bar{y}) \in H \text { and } h(y, \bar{y})+v \in U\} \text {. }
$$

Proof. The conjugate transform of $\left[\operatorname{Re} g^{+}(z, \bar{z}, K): G^{+}\right]$is given by (2) $\quad \sup \quad\left\{\operatorname{Re}\langle v, K)+\operatorname{Re}\langle y, z\rangle-\operatorname{Re} g^{+}(z, \bar{z}, k)\right\}$

$$
(z, \bar{z}, \kappa) \in G^{+} \quad \sup _{\kappa \in U^{*}}\left[\operatorname{Re}\langle v, K\rangle+\sup _{(z, \bar{z}) \in K G}\left\{\operatorname{Re}\langle y, z\rangle-\operatorname{Re} g^{+}(z, \bar{z}, \kappa)\right\}\right] .
$$

Explicit evaluation of equation (2) using Definitions 8 and 9 , and Lemma 1, gives a finite value of zero if

$$
h(y, \bar{y})+v \in-U .
$$

The primal formulation of a convex mathematical program in complex space is given below as Program A.

PROGRAM A. $\Phi=\inf \operatorname{Re} g_{0}\left(z_{0}, \bar{z}_{0}\right)+\sum_{j} \operatorname{Re} g_{j}^{+}\left(z_{j}, \bar{z}_{j}, \kappa_{j}\right)$ subject to the explicit constraints

$$
g_{i}\left(z_{i}, \bar{z}_{i}\right) \in-H_{i}, \quad i \in I,
$$

the implicit constraints

$$
\begin{gathered}
\left(z_{0}, \bar{z}_{0}\right) \in G_{0}, \\
\left(z_{i}, \bar{z}_{k}\right) \in G_{i}, \quad i \in I, \\
\left(z_{j}, \bar{z}_{j}, \kappa_{j}\right) \in G_{j}^{+}, j \in J,
\end{gathered}
$$

and the cone condition

$$
(z, \bar{z}) \in X
$$

where 


$$
\begin{aligned}
& z= z_{0} \underset{i \in I}{\times} z_{i} \underset{j \in J}{x} z_{j}, \\
& G_{j}^{+}=\left\{\left(z_{j}, \bar{z}_{j}, \kappa_{j}\right) \mid \kappa_{j}=0 \text { and } \sup _{y_{j} \in H_{j}} \operatorname{Re}\left\langle y_{j}, z_{j}\right\rangle<\infty\right\} \\
& \qquad\left\{\left(z_{j}, \bar{z}_{j}, \kappa_{j}\right\} \mid \kappa_{j} \in U_{j}^{*} \backslash\{0\} \text { and }\left(z_{j} / \kappa_{j}, \bar{z}_{j} / \bar{\kappa}_{j}\right) \in G_{j}\right\},
\end{aligned}
$$

and

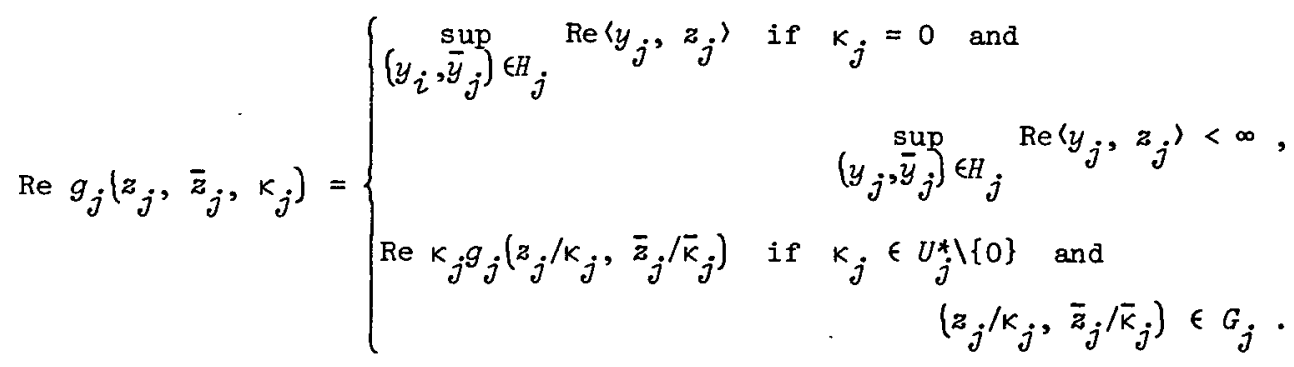

In Section 3, we show that the dual problem, Program $B$, corresponding to Program A is given by

$$
\text { PROGRAM B. } \Psi_{1}=\inf \operatorname{Re} h_{0}\left(y_{0}, \bar{y}_{0}\right)+\sum_{I} \operatorname{Re} h_{i}^{+}\left(y_{i}, \bar{y}_{i}, \lambda_{i}\right) \text { subject to }
$$

the explicit constraints

$$
h_{j}\left(y_{j}, \bar{y}_{j}\right) \in-U_{j}, \text { for all } j \in J,
$$

the implicit constraints

$$
\begin{gathered}
\left(y_{0}, \bar{y}_{0}\right) \in H_{0}, \\
\left(y_{i}, \bar{y}_{i}, \lambda_{i}\right) \in H_{i}^{+}, i \in I, \\
\left(y_{j}, \bar{y}_{j}\right) \in H_{j}, j \in J,
\end{gathered}
$$

and the cone condition

$$
(y, \bar{y}) \in x^{*}
$$

where

$$
\begin{aligned}
H_{i}^{+}=\left\{\left(y_{i}, \bar{y}_{i}, \lambda_{i}\right)\right. & \left.\mid \lambda_{i}=0 \text { and } \sup _{\left(z_{i}, \bar{z}_{i}\right\} \in G_{i}} \operatorname{Re}\left\langle y_{i}, z_{i}\right\rangle<\infty\right\} \\
& \cup\left\{\left(y_{i}, \bar{y}_{i}, \lambda_{i}\right) \mid \lambda_{i} \in W_{i}^{*} \backslash\{0\} \text { and }\left(y_{i} / \lambda_{i}, \bar{y}_{i} / \lambda_{i}\right) \in B_{i}\right\},
\end{aligned}
$$

and 


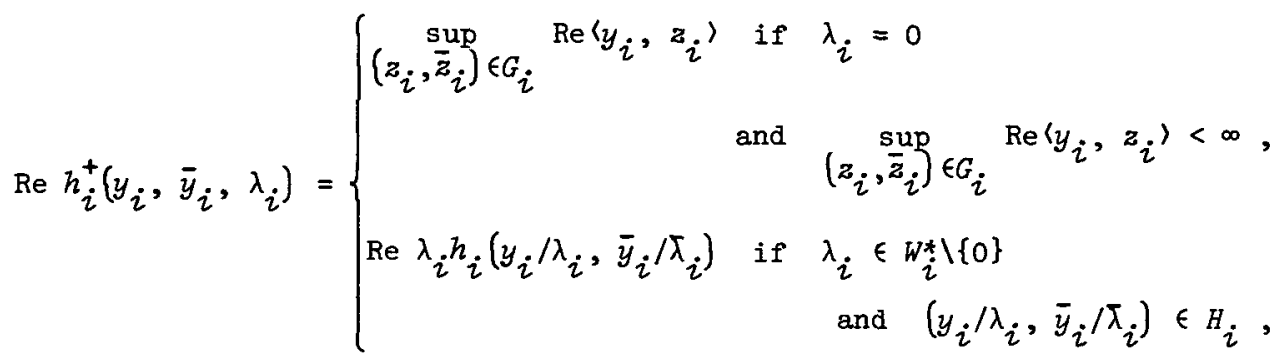

and $X^{*}$ is the polar cone of $X$.

We note that Programs A and B are completely symetric.

The primal and dual solutions are related by

$$
\begin{aligned}
& \left(z_{0}, \bar{z}_{0}\right) \in \partial h_{0}\left(y_{0}, \bar{y}_{0}\right), \\
& \left(z_{i}, \bar{z}_{i}\right) \in \partial h_{i}\left(y_{i} / \lambda_{i}, \bar{y}_{i} / \lambda_{i}\right), \lambda_{i} \in W_{i}^{*},
\end{aligned}
$$

and

$$
\left(z_{j} / \kappa_{j}, \bar{z}_{j} / \bar{\kappa}_{j}\right) \in \partial h_{j}\left(y_{j}, \bar{y}_{j}\right), \kappa_{j} \in U_{j}^{*} .
$$

Alternatively we have

$$
\begin{gathered}
\left(y_{0}, \bar{y}_{0}\right) \in \partial g_{0}\left(z_{0}, \bar{z}_{0}\right), \\
\left(y_{i} / \lambda_{i}, \bar{y}_{i} / \bar{\lambda}_{i}\right) \in \partial g_{i}\left(z_{i}, \bar{z}_{i}\right), \lambda_{i} \in W_{i}^{*}, \\
\left(y_{j}, \bar{y}_{j}\right) \in \partial g_{j}\left(\bar{z}_{j} / \bar{\kappa}_{j}\right), \kappa_{j} \in U_{j}^{*} .
\end{gathered}
$$

In addition, the primal and dual objective functions sum to zero (see Section 3 for the proof). Hence

$$
\Phi+\Psi=0 \text {. }
$$

These optimality conditions combined with feasibility conditions on the primal variables provide us with sufficient information to determine a primal optimal point from a dual optimal point.

\section{Duality theory}

From the construction of the conjugate transform and the lemmas of the previous section we have

$$
\operatorname{Re} g_{0}\left(z_{0}, \bar{z}_{0}\right)+\operatorname{Re} h_{0}\left(y_{0}, \bar{y}_{0}\right) \geq \operatorname{Re}\left(z_{0}, y_{0}\right)
$$


for feasible $\left(z_{0}, \bar{z}_{0}\right)$ and $\left(y_{0}, \bar{y}_{0}\right)$;

$$
\operatorname{Re} g_{j}^{+}\left(z_{j}, \bar{z}_{j}, \kappa_{j}\right)+0 \geq \operatorname{Re}\left\langle z_{j}, y_{j}\right\rangle
$$

for feasible $\left(z_{j}, \bar{z}_{j}\right),\left(y_{j}, \bar{y}_{j}\right)$, and for $j \in J$;

$$
0+\operatorname{Re} h_{i}^{+}\left(y_{i}, \bar{y}_{i}, \lambda_{i}\right) \geq \operatorname{Re}\left\langle z_{i}, y_{i}\right\rangle
$$

for feasible $\left(z_{i}, \bar{z}_{i}\right),\left(y_{i}, \bar{y}_{i}\right)$, and for $i \in I$.

Suming the equalities we get

(3) Re $g_{0}\left(z_{0}, \bar{z}_{0}\right)+\sum_{j \in J} \operatorname{Re} g_{j}^{+}\left(z_{j}, \bar{z}_{j}, \kappa_{j}\right)$

$$
+\operatorname{Re} h_{0}\left(y_{0}, \bar{y}_{0}\right)+\sum_{i \in I} \operatorname{Re} h_{i}^{+}\left(y_{i}, \bar{y}_{i}, \lambda_{i}\right) \geq \operatorname{Re}(z, y)=0,
$$

since $(z, \bar{z}) \in X$ and $(y, \bar{y}) \in \chi^{\perp}$.

THEOREM. Given Programs $\mathrm{A}$ and $\mathrm{B}$ are both consistent and the functions are closed convex, then

$\operatorname{Re} g_{0}\left(z_{0}, \bar{z}_{0}\right)+\sum_{j \in J} \operatorname{Re} g_{j}^{+}\left(z_{j}, \bar{z}_{j}, \kappa_{j}\right)$

$$
+\operatorname{Re} h_{0}\left(y_{0}, \bar{y}_{0}\right)+\sum_{i \in I} \operatorname{Re} h_{i}\left(y_{i}, \bar{y}_{i}, \lambda_{i}\right)=0 \text {, }
$$

for optimal $(z, \kappa)$ and $(y, \lambda)$.

Proof. By construction Re $g_{0}\left(z_{0}, \bar{z}_{0}\right)+\sum_{j \in J} \operatorname{Re} g_{j}^{+}\left(z_{j}, \bar{z}_{j}, \kappa_{j}\right)$ is convex in $(z, \bar{z}, \kappa)$ and $\operatorname{Re} h_{0}\left(y_{0}, \bar{y}_{0}\right)+\sum_{i \in I} \operatorname{Re} h_{i}^{+}\left(y_{i}, \bar{y}_{i}, \lambda_{i}\right)$ is convex in $(y, \bar{y}, \lambda)$. Suppose Program $B$ attains its minimum at a point $\left(y^{\prime}, \bar{y}^{\prime}, \lambda^{\prime}\right)$.

Consider the subgradient of $\operatorname{Re} h_{0}\left(y_{0}, \bar{y}_{0}\right)+\sum_{i \in I} \operatorname{Re} h_{i}\left(y_{i}, \bar{y}_{i}, \lambda_{i}\right)$ at the point $\left(y^{\prime}, \bar{y}^{\prime}, \lambda^{\prime}\right)$ and call it $\partial h\left(y^{\prime}, \bar{y}^{\prime}, \lambda^{\prime}\right)$. Now $\partial h\left(y^{\prime}, \bar{y}^{\prime}, \lambda^{\prime}\right) \subset G_{0} \underset{i \in I}{\times} G_{i} \underset{j \in J}{x} G_{j}^{+}$. If there exists $(z, \bar{z}, k) \in \partial h\left(y^{\prime}, \bar{y}^{\prime}, \lambda^{\prime}\right)$ which is feasible $((z, \bar{z}) \in \chi)$, then the left hand side of ( 3 ) is equal to zero. 
Suppose there does not exist $(z, \bar{z}, k) \in \partial h\left(y^{\prime}, \bar{y}^{\prime}, \lambda^{\prime}\right)$ such that $(z, \bar{z}) \in X$. Let $P\left(y^{\prime}, \bar{y}^{\prime}, \lambda^{\prime}\right)$ be the closed convex cone of feasible directions at $\left(y^{\prime}, \bar{y}^{\prime}, \lambda^{\prime}\right)$. Now $\partial h\left(y^{\prime}, \bar{y}^{\prime}, \lambda^{\prime}\right) \cap P\left(y^{\prime}, \bar{y}^{\prime}, \lambda^{\prime}\right)$ is a compact convex set and $X$ is a subspace. By the Hahn-Banach Theorem, there exists a function $\hat{y}$ such that $\operatorname{Re}(z, \hat{y})=0$ for $(z, \bar{z}) \in X$ and $\operatorname{Re}\langle z, \hat{y}\rangle<0$ for $(z, \bar{z}, \kappa) \in \partial h\left(y^{\prime}, \bar{y}^{\prime}, \lambda^{\prime}\right) \cap P\left(y^{\prime}, \bar{y}^{\prime}, \lambda^{\prime}\right)$.

This inplies that

$$
\left(y^{\prime}+\alpha y, \overline{y^{\prime}+\alpha \hat{y}}\right) \in x^{\perp} .
$$

Continuity tells us that there exists a small positive a such that

$$
\operatorname{Re} h_{0}\left(y_{0}, \bar{y}_{0}\right)+\sum_{i \in I} \operatorname{Re} h_{i}^{+}\left(y_{i}, \bar{y}_{i}, \lambda_{i}\right)
$$

at $\left(y^{\prime}+\alpha y, \overline{y^{\prime}+\alpha \hat{y}}, \lambda^{\prime}+\alpha \hat{\lambda}\right)$ is less than at $\left(y^{\prime}, \bar{y}^{\prime}, \lambda^{\prime}\right)$, which contradicts the optimality of $\left(y^{\prime}, \bar{y}^{\prime}, \lambda^{\prime}\right)$. The result follows.

\section{Example}

Consider the following problem:

$$
\begin{array}{ll}
\text { minimize } & \operatorname{Re}\left(\frac{1}{2} \bar{z} H_{0} z+h_{0} z+b_{0}+(\bar{z} A z)^{\frac{3}{2}}\right) \\
\text { subject to } & \frac{1}{2} \bar{z} H_{i} z+h_{i} z+b_{i} \in-W_{i}, \text { for all } i \in I,
\end{array}
$$

where $H_{i}, i \in I \cup\{0\}$, and $A$ are positive semidefinite hermitian $m \times m$ matrices, $h_{i}, i \in I \cup\{0\}$, and $b_{i}, i \in I \cup\{0\}$ are fixed column vectors in $C_{m}$ and scalars respectively, and $W_{i}, i \in I$, are convex polyhedral cones. Hence we are minimizing an objective which is part differentiable and part nondifferentiable subject to a finite number of quadratic constraints.

Since $H_{i}, i \in I \cup\{0\}$, and $A$ are positive semidefinite hermitian matrices, they may be decomposed into the product form

$$
H_{i}=\bar{D}_{i} D_{i}, \quad i \in I \cup\{0\} \text {, }
$$

and

$$
A=\bar{B} B
$$


With this decomposition, our problem may be cast in the separable form of Program A as

minimize $\quad \operatorname{Re}\left(\frac{\frac{1}{2}}{2} \bar{z}_{01} z_{01}+z_{02}+b_{0}+\left(\bar{z}_{03^{2}}\right)^{\frac{1}{2}}\right)$

subject to $\frac{f_{2}}{\bar{z}_{i 1}} z_{i 1}+z_{i 2}+b_{i} \in-W_{i}$, for all $i \in I$ and $(z, \bar{z}) \in \mathrm{X}$,

where $z=\left(z_{01}, z_{02}, z_{03}, z_{11}, \ldots, z_{12}, \ldots\right)^{T}$ and $z$ belongs to the

column space of the $n \times m$ matrix $\left(D_{0} h_{0} B D_{1} h_{1} D_{2} h_{2} \cdots\right)^{T}$, where

$T$ denotes the transpose.

For the dual program, Program B, we require the conjugate transform of quadratic functions, linear functions, and the nondifferentiable form $(\bar{z} z)^{\frac{3}{2}}$. We also require the dual space $x^{*}$ of $x$.

Since at optimality we have $\langle y, z\rangle=0$, the polar cone is

$$
x^{*}=\left\{(y, \bar{y}) \mid D_{0^{y}}{ }_{01}+h_{0} y_{02}+B y_{03}+\sum_{i \in I}\left(D_{i} y_{i 1}+h_{i} y_{i 2}\right)=0\right\} \text {. }
$$

We can now determine the dual program from the detailed prescription given in Program B. This is found to be:

$$
\begin{gathered}
\operatorname{Re}\left(\bar{y}_{01} \bar{y}_{01}-b_{0}\right)+\sum_{i \in I} \operatorname{Re}\left(\frac{3}{2} \lambda \bar{y}_{i 1} y_{i 1}+b_{i} \lambda_{i}\right) \\
\text { subject to } \lambda_{i} \in W_{i}^{*} \text { for all } i, \\
D_{0} y_{01}+h_{0}+B y_{03}+\sum_{i \in I}\left(D_{i} y_{i 1}+h_{i 2} y_{i 2}\right)=0, \\
\bar{y}_{03^{y}} y_{03} \leq 1, \\
y_{i 2}=\lambda_{i} e, \text { for all } i,
\end{gathered}
$$

where $e$ is a vector with components of unity.

It is now straightforward to generate the results of previous researchers by appropriate specification of given matrices ${ }_{i}, A$ vectors $h_{i}$, and scalars $b_{i}, i \in I \cup\{0\}$. 


\section{References}

[1] Robert A. Abrams, "Nonlinear programming in complex space: sufficient conditions and duality", J. Math. Anaz. App .38 (1972), 619-632.

[2] Robert A. Abrams and Adi Ben-Israel, "Nonlinear programming in complex space: necessary conditions", SIAM J. Control 9 (1971), 606-620.

[3] Adi Ben-Israel, "Linear equations and inequalities on finite dimensional, real or complex, vector spaces: a unified theory", J. Math. Anal. Appl. 27 (1969), 367-389.

[4] W. Fenchel, Convex cones, sets, and functions (Department of Mathematics Logistics Research Project, Princeton University, Princeton, New Jersey, 1953).

[5] Morgan A. Hanson and Bertram Mond, "Duality for nonlinear programming in complex space", J. Math. AnaZ. App Z. 28 (1969), 52-58.

[6] O.P. Jain and P.C. Saxena, "A duality theorem for a special class of programming problems in complex space", J. Optimization Theory App Z. 16 (1975), 207-220.

[7] Norman Levinson, "Linear programming in complex space", J. Math. Anaz. Appl. 14 (1966), 44-62.

[8] Bertram Mond, "Nonlinear nondifferentiable programing in complex space", Nonlinear programing, 385-400 (Proc. Sympos.

Mathematics Research Center, University Wisconsin, Madison, 1970. Academic Press, New York, London, 1970).

[9] Elmor L. Peterson, "Geometric programming", SIAM Rev. 18 (1976), $1-51$.

[10] R. Tyrrell Rockafellar, Convex conalysis (Princeton University Press, Princeton, New Jersey, 1970).

[11] Philip Wolfe, "A duality theorem for non-linear programming", Quart. Appt. Math. 19 (1961), 239-244.

School of Mechanical and Industrial Engineering, University of New South Wales, Kensington, New South Wales. 